PROCEEDINGS OF THE

AMERICAN MATHEMATICAL SOCIETY

Volume 126, Number 12, December 1998, Pages 3715-3720

S 0002-9939(98)04957-0

\title{
CYCLE RANK OF LYAPUNOV GRAPHS AND THE GENERA OF MANIFOLDS
}

\author{
R. N. CRUZ AND K. A. DE REZENDE \\ (Communicated by Linda Keen)
}

\begin{abstract}
We show that the cycle-rank $r(L)$ of a Lyapunov graph $L$ on a manifold $M$ satisfies: $r(L) \leq g(M)$, where $g(M)$ is the genus of $M$. This generalizes a theorem of Franks. We also show that given any integer $r$ with $0 \leq r \leq g(M), r=r(L)$ for some Lyapunov graph $L$ on $M, \operatorname{dim} M>2$.
\end{abstract}

\section{INTRODUCTION}

Let $M$ be a smooth, compact, connected $n$-manifold with boundary. The genus of $M, g(M),[1]$ is the maximal number of mutually disjoint, smooth, compact, connected, two-sided codimension one submanifolds that do not disconnect $M$. This definition coincides with the classical definition of genus of a compact orientable 2-manifold.

Let $f: M \rightarrow \mathbb{R}$ be a Lyapunov function associated to a flow and define the following equivalence relation on $M: x \sim_{f} y$ if and only if $x$ and $y$ belong to the same connected component of a level set of $f$. We call $M / \sim_{f}$ a Lyapunov graph. The cycle rank of a graph is the maximum number of edges that can be removed without disconnecting the graph.

We will generalize the following theorem of Franks [4].

Theorem 1.1. Let $\varphi_{t}$ be a smooth flow on a closed, connected $n$-manifold $M$ with Lyapunov function $f$. Let $L$ be the Lyapunov graph of $f$. Assume that $L$ is finite. If $M$ is orientable and $\beta_{1}(M)=0$ (the first Betti number), then the cycle-rank of $L, r(L)$, is 0 . Since $L$ is connected, this implies that $L$ is a tree.

We will show that $r(L) \leq g(M)$ if $L$ is a Lyapunov graph of a Lyapunov function $f$ associated to a smooth flow $\varphi_{t}$ on $M$. As a consequence of Corollary 2.4, which asserts that $\beta_{1}(M)=0$ if and only if $g(M)=0$, we prove Theorem 1.1 without the assumption: $M$ orientable. We will also prove that given an integer $r, 0 \leq r \leq g(M)$, there exists a Lyapunov graph $L$ associated to a Morse function $f$ with gradient flow $\varphi_{t}$ on $M$ such that $r(L)=r$.

Received by the editors January 22, 1997.

1991 Mathematics Subject Classification. Primary 58F09, 58F25; Secondary 57R65.

The second author was partially supported by Conselho Nacional de Desenvolvimento Científico e Tecnológico under Grant 300072/90.2 and Fundação de Amparo à Pesquisa do Estado de São Paulo.

(C) 1998 American Mathematical Society 


\section{The Genus of A MANifold}

The genus of a group $\pi, g(\pi)$, is the maximal rank $r$ of a free group $F_{r}(r$ is the number of generators) such that there exists an epimorphism $\pi \rightarrow F_{r}$. If $\pi$ is finitely presented, $g(\pi)<\infty$. In [1] Cornea proves the following theorem whose proof we sketch in Appendix A:

Theorem 2.1. Let $M$ be a smooth compact connected n-manifold with boundary. Then

1. $g(M) \leq g\left(\pi_{1} M\right)$,

2. $g(M)=g\left(\pi_{1} M\right)$ if $\partial M=\emptyset$.

By using the fact that a subgroup of a free group is free, Cornea [1] also proves the following theorem:

Theorem 2.2. Let $\pi_{1}, \pi_{2}$ be two finitely presented groups. Then $g\left(\pi_{1} * \pi_{2}\right)=$ $g\left(\pi_{1}\right)+g\left(\pi_{2}\right)$.

In [1], Cornea has the following result which we will prove here using $\mathbb{Z}$ coefficients.

Proposition 2.3. Let $M$ be a smooth, connected closed n-manifold. Then

1. $g(M) \leq \beta_{1}(M)$ (the first Betti number),

2. $\beta_{1}(M)>0 \Rightarrow g(M)>0$.

Proof. There is an epimorphism $h: \pi_{1} M \rightarrow F_{g}, F_{g}$ the free group on $g=g(M)=$ $g\left(\pi_{1} M\right)$ generators. We have the following commutative diagram:

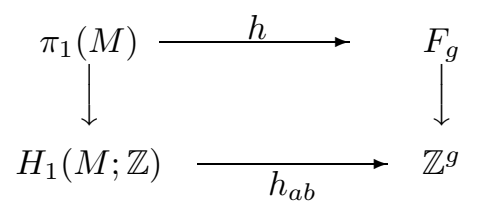

where the vertical maps are abelianization homomorphisms and $h_{a b}$ is $h$ abelianized. The homomorphism $h_{a b}$ is surjective because the other homomorphisms above are. Thus, result 1 . follows.

If $\beta_{1}(M)>0$, then $H_{1}(M ; \mathbb{Z})$ contains a direct summand isomorphic to $\mathbb{Z}$. In particular, there is an epimorphism $H_{1}(M ; \mathbb{Z}) \rightarrow \mathbb{Z}$. Composing this epimorphism with the abelianization $\pi_{1}(M) \rightarrow H_{1}(M ; \mathbb{Z})$ gives an epimorphism $\pi_{1}(M) \rightarrow \mathbb{Z}$. Thus, $g(M) \geq 1$.

Corollary 2.4. Let $M$ be a smooth, connected closed $n$-manifold. Then, $g(M)=0$ if and only if $\beta_{1}(M)=0$.

Proof. Straightforward.

Example 2.5. Let $\pi$ be an abelian group. Given an epimorphism $\pi \rightarrow F_{r}$, we conclude $r=0$ or 1 . Therefore, $g(\pi)=0$ or 1 . By the Structure Theorem for finitely generated abelian groups it follows that $g(\pi)=0$ if and only if $\pi$ is finite. Note that $g\left(\mathbb{Z}^{n}\right)=1$. Thus, we have the following examples:

1. $T^{n}=S^{1} \times \ldots \times S^{1}(n$ times $) g\left(T^{n}\right)=1$.

2. $g\left(\left(S^{1} \times S^{1} \times S^{2}\right) \#\left(S^{1} \times S^{3}\right) \# T^{4}\right)=3$.

3. $g\left(\left(T^{3} \times S^{2} \times S^{3}\right) \# T^{8} \#\left(T^{6} \times S^{2}\right) \#\left(S^{2} \times S^{6}\right)\right)=3$. 


\section{DyNAMiCs AND THE GENUS}

Proposition 3.1. Let $M$ be a connected, closed smooth n-manifold. Let $\varphi_{t}$ be a smooth flow on $M$ with associated Lyapunov function $f$. Let $L$ be a Lyapunov graph associated to $f$. Assume that $L$ is finite. Then

$$
r(L) \leq g(M)
$$

Proof. Set $r=r(L)$. Let $T \subset L$ be a maximal tree and $q: M \rightarrow L$ the quotient map. $L-T$ is the disjoint union of $r$ edges $e_{1}, \ldots, e_{r}$. By Sard's theorem it is possible to take points $x_{1}, \ldots, x_{r}, x_{1} \in e_{1}, \ldots, x_{r} \in e_{r}$ with the following properties: $q^{-1}\left(x_{1}\right), \ldots, q^{-1}\left(x_{r}\right)$ are submanifolds. These submanifolds are mutually disjoint, smooth, closed, connected, two-sided, codimension one submanifolds that do not disconnect $M$. The result follows.

Proposition 3.1 together with Corollary 2.4 provide a proof of Theorem 1.1 without the orientability assumption.

Example 3.2. Let $n$ be any large integer $>1, M=T^{n}$ and $L$ be the Lyapunov graph of a Lyapunov function $f$ associated to a smooth flow $\varphi_{t}$ on $T^{n}$. Proposition 3.1 implies that $r(L) \leq 1$. Thus, $L$ can have at most one cycle! However, the first Betti number of $T^{n}$ increases with $n$, therefore, genus, and not the first Betti number, is the right tool to study the cycle-rank of Lyapunov graphs.

Theorem 3.3. Let $M$ be a smooth, connected, closed n-manifold, $n>2$. Let $r$ be an integer with $0 \leq r \leq g(M)$. Then, there is a gradient flow $\varphi_{t}$ on $M$ with associated Morse function $f$ such that $r(L)=r, L$ the Lyapunov graph of $f$. In particular, $g(M)$ is the largest cycle-rank for Lyapunov graphs on $M$.

The fact that this theorem does not hold for $n=2$ is shown in [3].

Before we proceed with the proof, we will define a Lyapunov graph associated to a smooth flow on a smooth compact $n$-manifold with boundary $W$. Let $\varphi_{t}$ be a smooth flow on $W$ with associated Lyapunov function $\tilde{f}$. Let $\partial_{+} W$ be the component of $\partial W$ for which $\frac{\partial}{\partial t} \varphi_{t}$ points inward. Similarly, $\partial_{-} W$ is the component of $\partial W$ for which $\frac{\partial}{\partial t} \varphi_{t}$ points outward. The Lyapunov graph $\tilde{L}$ of $\tilde{f}$ is defined exactly as in [4]. However, since $\partial W \neq \emptyset$, the vertices correspond to components of the chain recurrent of $\varphi_{t}$ together with the boundary components of $W$. Vertices that correspond to boundary components will be referred to as boundary vertices. In what follows we assume $\tilde{L}$ is finite. We will need a lemma.

Lemma 3.4. Let $W$ be as above with $n>2$. Assume that $\tilde{f}$ is the Morse function associated to an ordered handle decomposition [5] $\tilde{\mathcal{H}}$ of $W$. In addition, assume $\partial_{+} W=\emptyset$ and $\tilde{\mathcal{H}}$ contains a single $n$-handle (equivalently we could assume $\partial_{-} W=\emptyset$ and $\mathcal{H}$ contains a single 0 -handle). Then, $\tilde{L}$ is a tree.

Proof (of Theorem 3.3). Let $N_{1}, \ldots, N_{r}, r \geq 1$, be mutually disjoint, smooth, closed, connected, two-sided codimension one submanifolds that do not disconnect $M$. Let $T_{1}, \ldots, T_{r}$ be mutually disjoint closed tubular neighborhoods of $N_{1}, \ldots, N_{r}$ respectively. Set $W=M-\bigcup_{i=1}^{r}$ int $T_{i}$. See Figure 1 . As $W$ is connected, we can choose an ordered handle decomposition $\widetilde{\mathcal{H}}$ for $(W ; \partial W, \emptyset)$ containing a single $n$ handle [5]. Choose handle decompositions $\mathcal{H}_{i}$ for $\left(T_{i} ; \emptyset, \partial T_{i}\right)$ which are ordered and 


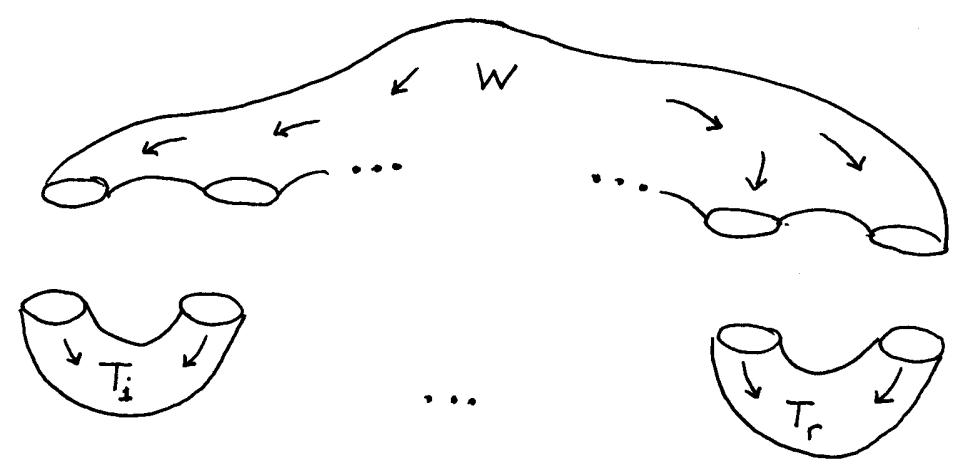

FiguRE 1. Decomposition of $M$.

contains just one 0 -handle, $i=1, \ldots, r$. Let $f_{i}, i=1, \ldots, r$, be Morse functions associated to these handle decompositions $\mathcal{H}_{i}$.

Define a handle decomposition $\mathcal{H}$ for $M$ as follows: $\mathcal{H}=\left(\bigcup_{i=1}^{r} \mathcal{H}_{i}\right) \cup \widetilde{\mathcal{H}}$. The order of attachment is: attach the handles of $\mathcal{H}_{1}$ respecting the order of an attachment of $\mathcal{H}_{1}$. Do the same for $\mathcal{H}_{2}, \ldots, \mathcal{H}_{r}, \widetilde{\mathcal{H}}$. Let $f$ be the Morse function that corresponds to $\mathcal{H}$, such that $\left.f\right|_{W}=\widetilde{f}$ and $\left.f\right|_{T_{i}}=f_{i}, i=1, \ldots, r$. Let $L$ be a Lyapunov graph of $f$ and $\widetilde{L}, L_{i}$ be Lyapunov graphs of $\widetilde{f}, f_{i}, i=1, \ldots, r . L$ is obtained by "glueing" $\widetilde{L}$ and $L_{i}, i=1, \ldots, r$, along boundary vertices that correspond to the $2 r$ components of $\partial W$ and to the two components of $\partial T_{i}, i=1, \ldots, r$. Since $\partial W$ has $2 r$ components and $\widetilde{\mathcal{H}}$ is an ordered handle decomposition with one $n$-handle, then by Lemma 3.4 the corresponding graph $\tilde{L}$ is a tree as shown in Figure 2. Similarly, $\partial T_{i}$ has 2 components and the handle decomposition $\mathcal{H}_{i}$ is ordered and has one 0-handle. Hence, by Lemma 3.4 the corresponding graph $L_{i}$ is a tree as shown below in Figure 2. The "glueing" of the graphs will be performed so that the edge labellings incident to a pair of boundary vertices that will be identified match. Furthermore, a pair of identified boundary vertices becomes an edge point (more precisely, if $e_{-}, e_{+}$are edges incident to a boundary vertex $v, e=e_{-} \cup\{v\} \cup e_{+}$is the edge in $L$ for which $v$ is an edge point). Hence, $r(L)=r$. We now prove the case $r=0$. In view of a result of Smale's [6] we can take an ordered Morse function $f$ on $M$ with one index 0 singularity and one index $n$ singularity. By results in [2] as summarized in Table 1, all level sets are connected and the graph $L$ associated to the flow is linear. Hence $r(L)=0$.

Proof (of Lemma 3.4). Assume $\partial_{+} W=\emptyset$. The case $\partial_{-} W=\emptyset$ is analogous. $L$ is oriented by the direction of flow lines. Let $v$ be a vertex of $L$. Denote by $e^{-}(v), e^{+}(v)$ the number of outgoing, ingoing edges of $v$, respectively. If $v$ corresponds to a component of $\partial_{-} W$, then $e^{-}(v)=0, e^{+}(v)=1$. If $v$ corresponds to an index $i$ singularity of $\varphi_{t}$, we set ind $v=i$ (the index of $v$ ). By Corollary 3.1 in [2] we have:

$L$ is path-connected because $W$ is. Let $v_{n}$ be the unique vertex with ind $v_{n}=n$. Since we are assuming an ordered handle decomposition, this implies that $e^{+}(v)=1$ if $v$ is a vertex with ind $v=n-1$. 

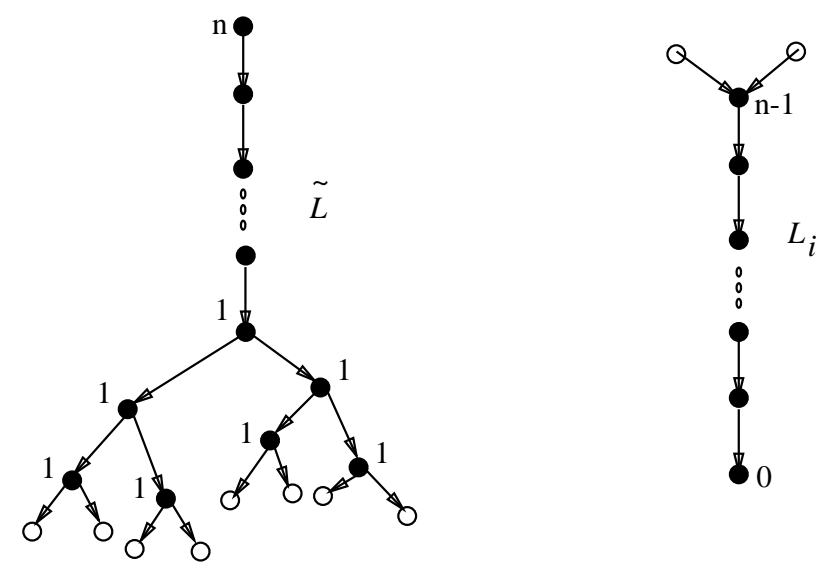

Figure 2. Lyapunov graphs $\tilde{L}$ and $L_{i}$. Here, 'indicates a vertex labelled with the index of a singularity of $\varphi_{t}$ and "O" indicates a boundary vertex.

TABLE 1

\begin{tabular}{|c|c|c|c|c|c|}
\hline ind $v$ & 0 & 1 & $1<i<n-1$ & $n-1$ & $n$ \\
\hline$e^{-}(v)$ & 0 & 1 or 2 & 1 & 1 & 1 \\
\hline$e^{+}(v)$ & 1 & 1 & 1 & 1 or 2 & 0 \\
\hline
\end{tabular}

Otherwise, if $e^{+}(v)=2$ we would necessarily have to attach an index $n-1$ handle before attaching an index 1 handle. The orientation of $L$ is equivalent to a partial order relation for its vertices such that $v \leq u$ if $\partial e=\{v, u\}, e$ an edge which is ingoing for $v$ (equivalently $e$ is outgoing for $u$ ). We define $\partial^{+} e=v$ and $\partial^{-} e=u$.

Let $\mathcal{V}, \mathcal{E}$ be the vertices and edges of $L$ respectively. By the previous table and paragraph, $e^{+}(v)=1$ for all $v \in \mathcal{V}-\left\{v_{n}\right\}$. This fact allows us to define a bijection $\mathcal{F}: \mathcal{V}-\left\{v_{n}\right\} \rightarrow \mathcal{E}$ as follows: for $v \in \mathcal{V}-\left\{v_{n}\right\}, \mathcal{F}(v)=e$ where $\partial^{+} e=v$, i.e., $e$ is the edge ingoing for $v$. Conclusion: the Euler characteristic of $L$ is one. Thus, $L$ is a tree.

\section{Appendix A}

We sketch here a proof of the following theorem of Cornea's [1]:

Theorem 3.5. Let $M$ be a smooth compact connected n-manifold with boundary. Then

1. $g(M) \leq g\left(\pi_{1} M\right)$,

2. $g(M)=g\left(\pi_{1} M\right)$ if $\partial M=\emptyset$.

Proof. (Sketch) Part 1. is proved as follows. Let $N_{1}, \ldots, N_{r}, r \leq g(M)$ (at this point we do not know that $g(M)<\infty$ ), be mutually disjoint, smooth, compact, connected, two-sided codimension one submanifolds that do not disconnect $M$. Let $T_{1}, \ldots, T_{r}$ be mutually disjoint closed tubular neighborhoods of $N_{1}, \ldots, N_{r}$ respectively. There are diffeomorphisms of pairs $\varphi_{i}:\left(T_{i}, N_{i}\right) \rightarrow\left(N_{i} \times D^{1}, N_{i} \times\right.$ 
$\{0\}), i=1, \ldots, r$. Collapsing $W=M-\bigcup_{i=1}^{r} T_{i}$ to a point and $T_{i}$ to $\left\{x_{i}\right\} \times D^{1}, x_{i} \in$ $N_{i}, i=1, \ldots, r$, defines an equivalence relation $\sim$ on $M$. Hence $M / \sim=B_{r}$, a bouquet of $r$ circles. Let $c: M \rightarrow B_{r}$ be the quotient map. The circles of $B_{r}$ are $c\left(\varphi_{i}^{-1}\left(\left\{x_{i}\right\} \times D^{1}\right)\right), i=1, \ldots, r$. As $W$ is path-connected, it follows that $c_{*}: \pi_{1}(M) \rightarrow \pi_{1}\left(B_{r}\right) \cong F_{r}$ is an epimorphism. Conclusion: $r \leq g\left(\pi_{1} M\right)$. As $g(M)$ is the maximal number of mutually disjoint, smooth, compact, connected, two-sided codimension one submanifolds that do not disconnect $M$, it follows that $g(M) \leq g\left(\pi_{1} M\right)(<\infty)$.

Part 2. is proved as follows. Let $g=g\left(\pi_{1} M\right)$. Start with an epimorphism $\pi_{1} M \rightarrow \pi_{1}\left(B_{g}\right) \cong F_{g}$. As $B_{g}$ is an Eilenberg-Mac Lane space (see [7], p. 225), the above epimorphism is induced by a continuous map $h: M \rightarrow B_{g}$. The basepoint of $B_{g}, b$, is the intersection of all circles of $B_{g}$. Let $x_{0}$ be any point of $M$. We may assume that the pre-image of $b$ is $x_{0}$. Thus, $h$ is the one point union of $g$ maps from $M$ to circles containing $b$. Homotopying rel $x_{0}$ if necessary, we may assume that these maps are smooth. Now, by Sard's theorem there are points $b_{1}, \ldots, b_{g} \in B_{g}-\{b\}$ lying in disjoint circles which are regular values for the functions that make up $h$. Conclusion: $h^{-1}\left(b_{1}\right), \ldots, h^{-1}\left(b_{g}\right)$ are mutually disjoint, smooth, closed, connected codimension one submanifolds that do not disconnect $M$. Thus, $g\left(\pi_{1} M\right) \leq g(M)$. Part 2. follows.

\section{REFERENCES}

[1] O. Cornea, The genus and the fundamental group of high-dimensional manifolds, Stud. Cerc. Mat. 41 (1989), no. 3, 169-178. MR 90h:57022

[2] R. Cruz and K. de Rezende, Gradient-like flows on high-dimensional manifolds, to appear.

[3] K. de Rezende and R. D. Franzosa, Lyapunov Graphs and Flows on Surfaces, Trans. of the Amer. Math. Soc., 340, (1993), 767-784. MR 94b:58086

[4] J. Franks, Non-singular Smale flows on $S^{3}$, Topology 24, (1985), 265-282. MR 87d:58110

[5] J. Milnor, Lectures on the h-cobordism theorem, Annals of Math. Studies, Princeton Univ. Press, Princeton, N.J, 1965. MR 32:8352

[6] S. Smale, Generalized Poincaré Conjecture in dimensions greater than four, Annals of Math., 74, (1961), 391-406. MR 25:580

[7] G. Whitehead, Homotopy Theory. Springer, 1978. MR 80b:55001

Departamento de Matemática, Universidade Estadual de Campinas, 13083-970 CampiNAS, SÃo PaUlo, Brazil

E-mail address: cruz@turing.unicamp.br

E-mail address: ketty@ime.unicamp.br 